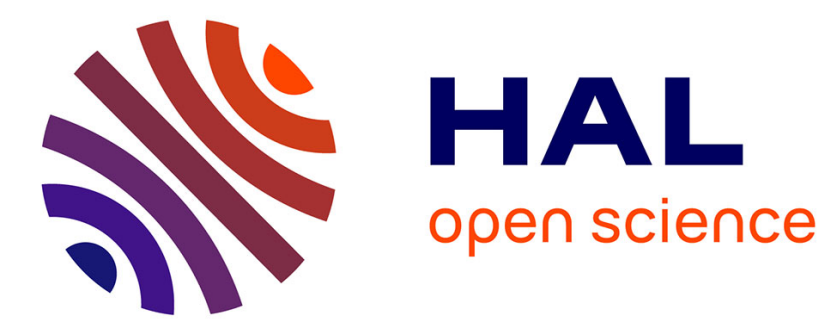

\title{
Two-orders fast multipole analysis of meta-atoms
}

Geoffroy Klotz, Nicolas Mallejac, Stefan Enoch

\section{To cite this version:}

Geoffroy Klotz, Nicolas Mallejac, Stefan Enoch. Two-orders fast multipole analysis of meta-atoms. ICEAA 2019, Sep 2019, Grenade, France. hal-02273852

\section{HAL Id: hal-02273852 \\ https://hal.science/hal-02273852}

Submitted on 29 Aug 2019

HAL is a multi-disciplinary open access archive for the deposit and dissemination of scientific research documents, whether they are published or not. The documents may come from teaching and research institutions in France or abroad, or from public or private research centers.
L'archive ouverte pluridisciplinaire HAL, est destinée au dépôt et à la diffusion de documents scientifiques de niveau recherche, publiés ou non, émanant des établissements d'enseignement et de recherche français ou étrangers, des laboratoires publics ou privés. 


\section{Two-orders fast multipole analysis of meta-atoms}

\author{
Geoffroy Klotz \\ CEA/DAM Le Ripault \\ CEA \\ Monts, France \\ geoffroy.klotz@cea.fr
}

\author{
Nicolas Malléjac \\ CEA/DAM Le Ripault \\ CEA \\ Monts, France \\ nicolas.mallejac@cea.fr
}

\author{
Stefan Enoch \\ Institut Fresnel \\ Aix Marseille Université \\ Marseille, France \\ stefan.enoch@fresnel.fr
}

\begin{abstract}
Meta-materials are complex structured media that are designed in order to get unusual physical properties that often cannot be reached in nature. In the field of electromagnetic, many interesting research topics of the past years are based on our ability to create these specific materials that would behave as no preexisting natural materials. Meta-materials are designed in order to mimic a needed medium that we can call the "targeted material": most of the time, targeted materials are regular theoretical magneto-dielectrics, whose electromagnetic behavior is encoded into two property tensors: a permittivity tensor that describes the response to an electric field, and a permeability tensor that describes the response to a magnetic field. One of the most popular application involving meta-materials is the superlens, that requires a negative refraction index: both negative permittivity and permeability are needed to get a negative refraction in the lens. Invisibility cloaking and routing are others well known applications, that need a rigorous control of gradient properties of both permittivity tensor and permeability tensor in the medium.

Theoretically, a magneto-dielectric is composed of an assembly of small dipoles: the use of resonant devices playing the role of the dipoles allow researchers to reach almost every targeted values of permittivity and permeability. However, these resonances are complex and homogenization of arrays of such inclusions is challenging: spatial dispersion, bianisotropy, high-order multipolar moments and coupling effects contribute to move the reality away from the model of a hypothetical assembly of dipoles. In our researches, we try to develop meta-materials that would behave as there target for any incoming electromagnetic field and environment. Multipole decomposition is a powerful tool that helps in the study of scatterers, and especially for meta-atoms in meta-materials. We developed an algorithm that performs a fast two-orders multipole expansion based on the extraction of the far field in six different directions. A comparison of the scattered field of the simulated inclusion with the scattered field of the twoorders multipole model is performed, and informs us about the degree of precision of the model. This approach appears to be efficient in the design of meta-materials intended for cloaking applications.
\end{abstract}

Index Terms-homogenization, metamaterials, multipole expansion, electromagnetism, permittivity, permeability

\section{INTRODUCTION}

Homogenization extracts continuous macroscopic physical properties of micro-structured meta-materials. It is highly challenging and requires a good understanding of the underlying physics. Homogenization has been topical in physics and applied mathematics since the beginning of the 20th Century [1] [2] [3] resulting in a rich scientific literature. In order to extract permittivity and permeability properties of an electromagnetic metamaterial, classical retrieval procedure such as the Nicolson Ross method have been investigated [4] : this method suffers from a limitation over the sample thickness, that should be under $\lambda / 2$. Alternative methods based on Nicolson and Ross extraction have been investigated to extend it to sample thickness that are larger than the half wavelength [5] [6]. Another approach for homogenization is based on the analysis of the near electromagnetic field in the material itself [7] [8] : effective parameters are determined by averaging of the electric and magnetic fields in the unit cell.

Using standard procedures such as Nicolson Ross, one can always compute effective permittivity and permeability for a given meta-material. However, the computed effective parameters may lead to a weak homogeneous model of the metamarial and not accurately represent its physical behaviour in a different context. Indeed, interactions and coupling between electric and magnetic response of scatterers are responsible for complex phenomenon. To give a physical meaning to homogenization, a method consists in considering that metamaterials are bianisotropic media. This approach is exploited in [9] [10] [11] and the issue is also addressed in [7].

A theoretical magneto-dielectric material is an assembly of microscopic electric and magnetic dipoles. That point is already an issue, because most of the classical resonating inclusions in litterature are neither dipoles nor microscopic. Indeed, resonance wavelength is linked to the size of the inclusion : a regular wire sees its first resonance at $\lambda / 2$, as a half wave antenna does. A split ring resonator (SRR) can resonate down to $\lambda / 10$. In this paper, we have in mind applications such as cloaking, where three-dimensions metamaterials are needed. An approach of homogenization that provides physical insight is required to obtain a meta-material that would behave as the targetted magneto-dielectric for any incoming electromagnetic fields and environment.

\section{Methodology}

As a first step we look for a proper method to characterize scatterers in the far field. Multipole decomposition is a powerful tool that helps in the study of scatterers, and especially for meta-atoms in meta-materials [12] [13] [14]. This method leads to very interesting results and gives clues to the understanding of phenomena involved when studying resonating scatterers.

The main limit with this method is its complexity and computational burden. In [12] for example, 10000 evaluation points are used to perform the multipole decomposition. 
In this paper, we propose a fast method that gives a two-orders multipole expansion of any scatterer by exploiting the far field. The electric field radiated by an electric two-orders multipole is given by the sum of the dipole and quadrupole contributions, as detailed in [15]:

$\mathbf{E}_{\mathbf{e}}=\frac{Z_{0} c k^{2}}{4 \pi}((\mathbf{n} \times \mathbf{p}) \times \mathbf{n}) \frac{e^{i k r}}{r}-\frac{i Z_{0} c k^{3}}{24 \pi}\left(\left(\mathbf{n} \times \mathbf{Q}_{\mathbf{e}}(\mathbf{n})\right) \times \mathbf{n}\right) \frac{e^{i k r}}{r}$

where $\mathbf{n}$ is the unit vector pointing in the direction of observation, $\mathbf{p}$ is the electric dipole moment and $\mathbf{Q}_{\mathbf{e}}(\mathbf{n})$ is a vector defined in Eq.4 which encapsulates the quadrupolar moments. Electric field radiated by a magnetic multipole takes a slightly different form :

$$
\mathbf{E}_{\mathbf{m}}=\frac{-Z_{0} k^{2}}{4 \pi}(\mathbf{n} \times \mathbf{m}) \frac{e^{i k r}}{r}+\frac{i Z_{0} k^{3}}{24 \pi}\left(\left(\mathbf{n} \times \mathbf{Q}_{\mathbf{m}}(\mathbf{n})\right) \frac{e^{i k r}}{r}\right.
$$

We assume that our scatterer is small enough to consider that the source can be represented by a point. If higher orders are negligible, the total far field scattered by the equivalent point source is given by :

$$
\begin{gathered}
\mathbf{E}_{\mathbf{s c a t}, \mathbf{f a r}}=\frac{-Z_{0} k^{2}}{4 \pi} \frac{e^{i k r}}{r} \mathbf{n} \times\left(\mathbf{m}-\frac{i k}{6} \mathbf{Q}_{\mathbf{m}}(\mathbf{n})\right) \\
+\frac{Z_{0} c k^{2}}{4 \pi} \frac{e^{i k r}}{r} \mathbf{n} \times\left(\mathbf{p}-\frac{i k}{6} \mathbf{Q}_{\mathbf{e}}(\mathbf{n})\right) \times \mathbf{n}
\end{gathered}
$$

with $\mathbf{Q}_{\mathbf{e}}(\mathbf{n})$ and $\mathbf{Q}_{\mathbf{m}}(\mathbf{n})$ depending on respectively the electric quadrupolar moment tensor $Q e_{\alpha \beta}$ and the magnetic quadrupolar moment tensor $Q m_{\alpha \beta}$ :

$$
\begin{aligned}
& \mathbf{Q}_{\mathbf{e}}(\mathbf{n})=\sum_{\beta} Q e_{\alpha \beta} n_{\beta} \\
& \mathbf{Q}_{\mathbf{m}}(\mathbf{n})=\sum_{\beta} Q m_{\alpha \beta} n_{\beta}
\end{aligned}
$$

We want to determine both dipolar and quadrupolar moments. Quadrupolar tensors are 3-by-3 symetric matrix and their diagonal coefficients are equal to zero: only three unknown variables are needed to know entirely each quadrupolar moment tensor. Dipolar moments are 3-by-1 vectors which means that we have 12 unknown quantities to find in order to characterize properly the scatterer. We evaluate the electric far field for a given direction defined by its angular coordinates $(\theta, \phi)$. To have a simple example we choose the $(0,0)$ direction, that is equivalent to the $\mathrm{z}$-axis.

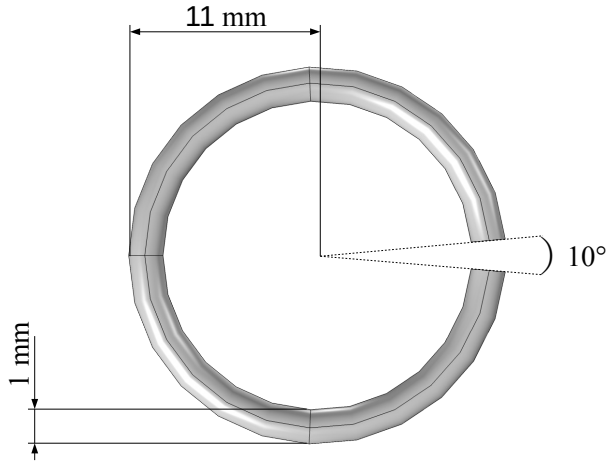

Fig. 1. Geometry of the metallic Split-Ring Resonator (SRR) we simulated with COMSOL Multiphysics: this a split torus which external curvilinear length equals $69 \mathrm{~mm}$. The incoming plane wave in the simulation is traveling from the left to the right, and the magnetic field is oriented perpendicularly to the cut plane of the figure.

$$
\begin{cases}\mathbf{E}_{\mathbf{s c a t}, \mathbf{f a r}}(0,0) \cdot \mathbf{x}=\quad & \frac{-Z_{0} k^{2}}{4 \pi} \frac{e^{i k r}}{r}\left(m_{y}-\frac{i k}{6} Q m_{x z}\right) \\ & +\frac{Z_{0} c k^{2}}{4 \pi} \frac{e^{i k r}}{r}\left(p_{x}-\frac{i k}{6} Q e_{x z}\right) \\ \mathbf{E}_{\mathbf{s c a t}, \mathbf{f a r}}(0,0) \cdot \mathbf{y}=\quad \frac{-Z_{0} k^{2}}{4 \pi} \frac{e^{i k r}}{r}\left(-m_{x}+\frac{i k}{6} Q m_{y z}\right) \\ \\ +\frac{Z_{0} c k^{2}}{4 \pi} \frac{e^{i k r}}{r}\left(p_{y}-\frac{i k}{6} Q e_{y z}\right) \\ \mathbf{E}_{\mathbf{s c a t}, \mathbf{f a r}(0,0) \cdot \mathbf{z}=0}\end{cases}
$$

We obtain in Eq.5 two non-trivial equations along the $\mathrm{x}$ and $\mathbf{y}$ directions. Given that we study only the far field, it is normal to find that the projection along vector $\mathbf{z}$, also direction of observation, is equal to zero. The $\mathrm{z}$ component of the electric field does not propagate along $\mathbf{z}$ direction. One evaluation point gives us two equations implying eight different unknown variables. To obtain enough equations, six evaluation directions at least are needed. The whole system of equations can then be represented in the single matrix equation Eq.6.

$$
\frac{4 \pi r e^{-i k r}}{Z_{0} k^{2}} \mathbf{E}=M . \mathbf{X}
$$

where :

- $\mathbf{E}$ is a 12-by-1 vector composed of the non-trivial projections along $\mathbf{x}, \mathbf{y}$ or $\mathbf{z}$ of the complex electric far field measured in the 6 directions of evaluation (two projections per direction);

- $\mathrm{M}$ is an invertible 12-by-12 matrix that depends on the chosen set of directions ;

- $\mathbf{X}$ is a 12-by-1 vector composed of the 12 unknown variables of the system : $p_{\mu}, m_{\nu},-\frac{i k}{6} Q e_{\mu \nu}$ and $-\frac{i k}{6} Q m_{\mu \nu}$ 

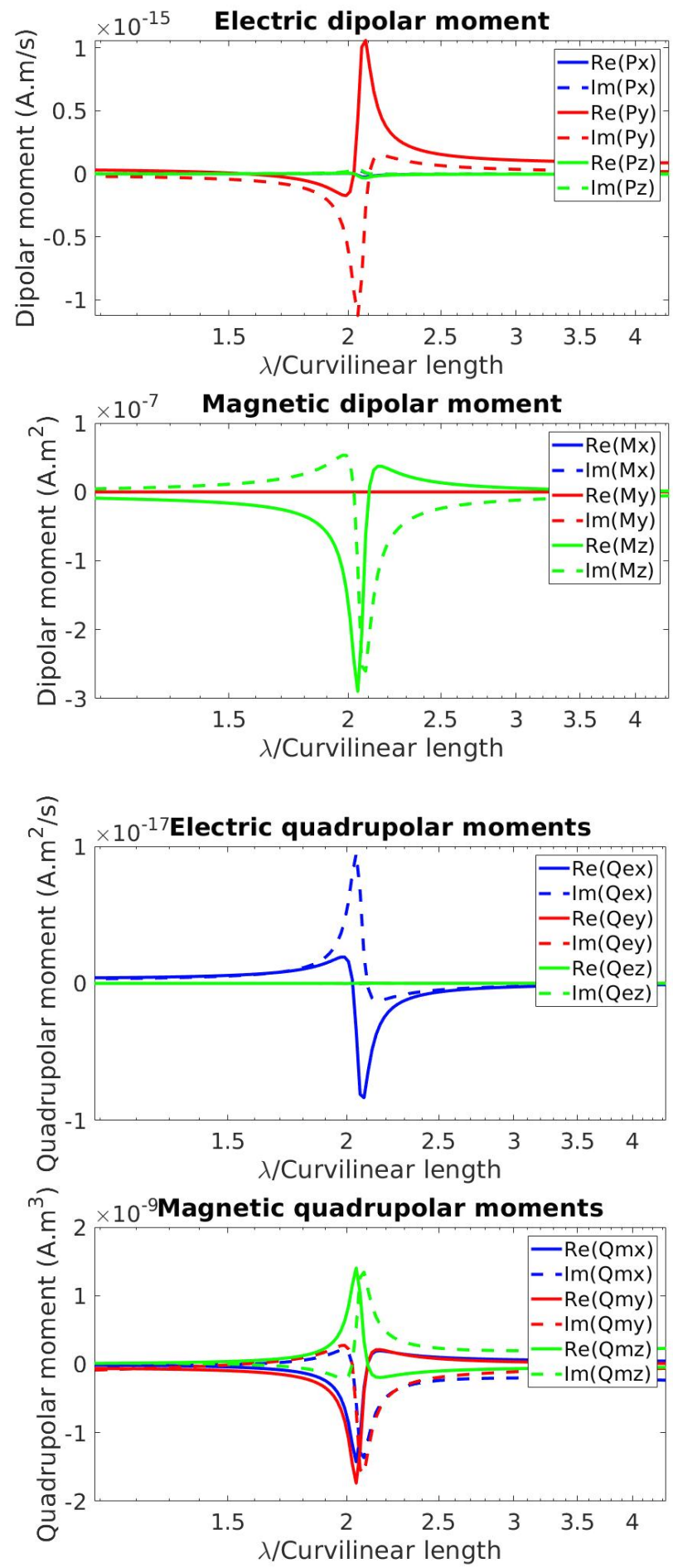

Fig. 2. Electric and magnetic moments of the first and second order for a classical Split-Ring Resonator (SRR). The length of the incoming wave is $\lambda$, and the curvilinear length of the SRR is $69 \mathrm{~mm}$. Calculations of these moments are based on the method presented in this paper.
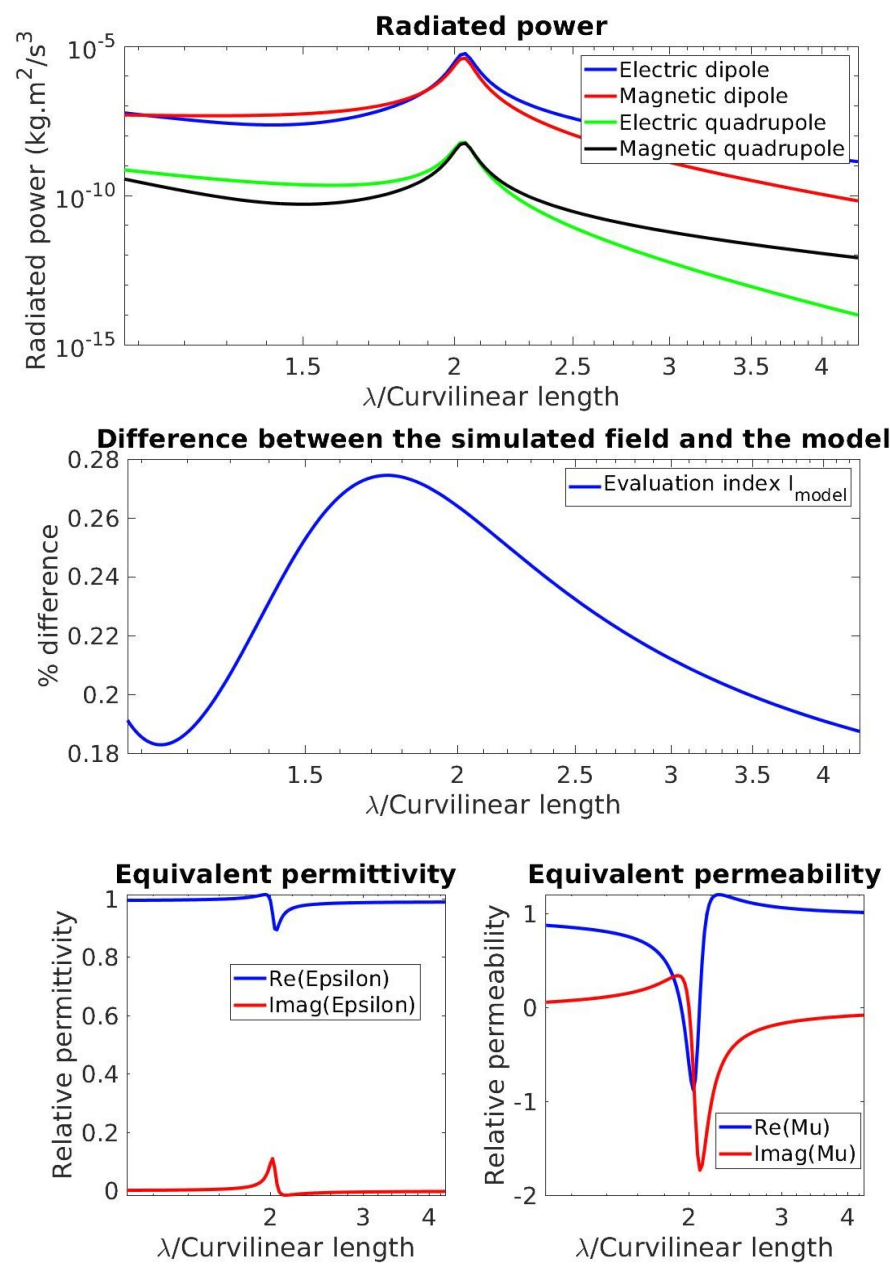

Fig. 3. Power radiated for every moment of the multipole expansion. An integral criterion defined in Eq.7 compares the two-orders model and the simulated scattered field : at the resonance, for $\lambda /$ Curvilinear length $=2.05$, the difference is only of $0.25 \%$. The equivalent permittivity and permeability are estimated via the Clausius-Mossotti relation, assuming a 3D-lattice of SRR with $1 \mathrm{~cm}$ spacing between each neighbour.

To solve that system, a simple inversion of the matrix $M$ is required. $M$ only depends on the set of evaluation directions we chose, and would be different for another set. Every inclusion exhibits its proper vector $\mathbf{E}$ that can be evaluated using either a full wave simulation or an experimental measurement. For this paper, we performed simulations with the commercial software COMSOL Multiphysics, using the RF module. Any design of inclusion can be tested thanks to that method : the following calculations are made for a simulation of a classic Split-Ring Resonator (SRR) which geometric properties are given in Fig.1 We simulated the metallic SplitRing Resonator in free space, lightened by an incoming plane wave which magnetic field was oriented perpendicularly to the cut plane of Fig.1. Results are given on Fig.2. To validate our approach, we compare the scattered simulated field and the field scattered by the 2-orders multipole model, using the evaluation index $I_{\text {model }}$ defined in equation Eq.7. An evaluation of this efficiency index is also represented on Fig.2. 


$$
I_{\text {model }}=\frac{\iint_{\theta, \phi}\left\|\mathbf{E}_{\text {model }}-\mathbf{E}_{S R R}\right\|^{2} \sin (\theta) d \theta d \phi}{\iint_{\theta, \phi}\left\|\mathbf{E}_{\text {model }}\right\|^{2} \sin (\theta) d \theta d \phi}
$$

As we now know the dipolar response of a single SRR in free space, it is possible to estimate the equivalent permittivity and permeability of an array of such devices thanks to the Clausius-Mossotti relation.

$$
\epsilon_{y y}(\omega)=\frac{1+\frac{2 p_{y}(\omega)}{3 V \epsilon_{0} E_{b k g d}}}{1-\frac{p_{y}(\omega)}{3 \epsilon_{0} V E_{b k g d}}} \quad ; \quad \mu_{z z}(\omega)=\frac{1+\frac{2 \mu_{0} m_{z}(\omega)}{3 V H_{b k g d}}}{1-\frac{\mu_{0} m_{z}(\omega)}{3 V H_{b k g d}}}
$$

with $p(\omega)$ and $m(\omega)$ the electric and magnetic dipolar moments, $E_{b k g d}$ and $H_{b k g d}$ the amplitude of the electric and megnetic background fields and $V$ the volume of the unit cell containing the SRR. Results are given on Fig.3. This approach gives an estimation of the effective properties of a metamaterial composed of such resonating structure thanks to the study of the field radiated by only one of them.

\section{CONCLUSION}

The method presented in this manuscript is very convenient to study small inclusions for meta-materials, and helps in the understanding of the coupling effects between surface currents and an incoming plane wave. It shows good results for small inclusions because of the negligible amplitude of high orders multipoles in such devices. After the lowest frequency resonance, efficiency of our approach deteriorates rapidly as frequency increases, because of higher orders that are not taken into account. Of course, the six-points evaluation could be improved in order to take into account higher orders of the multipole expansion. If the dipolar moments are overriding, an approximation of the permittivity and permeability of a lattice can be estimated via the Clausius-Mossotti relations.

\section{ACKNOWLEDGMENT}

This work and related research of G.K., N.M. and S.E. are funded by the french Direction Generale de l'Armement (DGA) and by the french Commissariat à l'Energie Atomique et aux Energies Alternatives (CEA).

\section{REFERENCES}

[1] J. C. Maxwell Garnett and Larmor Joseph. Colours in metal glasses and in metallic films. Philosophical Transactions of the Royal Society of London. Series A, Containing Papers of a Mathematical or Physical Character, 203(359-371):385-420, January 1904.

[2] D. a. G. Bruggeman. Berechnung verschiedener physikalischer Konstanten von heterogenen Substanzen. I. Dielektrizitätskonstanten und Leitfähigkeiten der Mischkörper aus isotropen Substanzen. Annalen der Physik, 416(7):636-664, 1935.

[3] Graeme W. Milton. The Theory of Composites. Cambridge University Press, Cambridge, 2002.

[4] A. M. Nicolson and G. F. Ross. Measurement of the Intrinsic Properties of Materials by Time-Domain Techniques. IEEE Transactions on Instrumentation and Measurement, 19(4):377-382, November 1970.
[5] O. Luukkonen, S. I. Maslovski, and S. A. Tretyakov. A Stepwise Nicolson-Ross-Weir-Based Material Parameter Extraction Method. IEEE Antennas and Wireless Propagation Letters, 10:1295-1298, 2011.

[6] A. L. de Paula, M. C. Rezende, and J. J. Barroso. Modified NicolsonRoss-Weir (NRW) method to retrieve the constitutive parameters of lowloss materials. In 2011 SBMO/IEEE MTT-S International Microwave and Optoelectronics Conference (IMOC 2011), pages 488-492, October 2011.

[7] David R. Smith and John B. Pendry. Homogenization of metamaterials by field averaging (invited paper). JOSA B, 23(3):391-403, March 2006.

[8] J.-M. Lerat, N. Malléjac, and O. Acher. Determination of the effective parameters of a metamaterial by field summation method. Journal of Applied Physics, 100(8):084908, October 2006.

[9] O. Ouchetto, C. Qiu, S. Zouhdi, L. Li, and A. Razek. Homogenization of 3-D Periodic Bianisotropic Metamaterials. IEEE Transactions on Microwave Theory and Techniques, 54(11):3893-3898, November 2006.

[10] M. C. K. Wiltshire, J. B. Pendry, and J. V. Hajnal. Chiral Swiss rolls show a negative refractive index. Journal of Physics: Condensed Matter. 21(29):292201, June 2009.

[11] Andrea Alù. Restoring the physical meaning of metamaterial constitutive parameters. Physical Review B, 83(8), February 2011.

[12] Stefan Mühlig, Christoph Menzel, Carsten Rockstuhl, and Falk Lederer. Multipole analysis of meta-atoms. Metamaterials, 5(2):64-73, June 2011.

[13] David J. Cho, Feng Wang, Xiang Zhang, and Y. Ron Shen. Contribution of the electric quadrupole resonance in optical metamaterials. Physical Review B, 78(12):121101, September 2008.

[14] Yong Zeng, Colm Dineen, and Jerome V. Moloney. Magnetic dipole moments in single and coupled split-ring resonators. Physical Review $B$, 81(7):075116, February 2010.

[15] John David Jackson. Classical Electrodynamics. 1962. 